\title{
SUPPLEMENTATION AND OPTIMIZATION OF GROUNDNUT SHELL IN THE DIET OF CONFINED UNSEXED MIXED CATTLE BREED DURING THE WINTER SEASON IN NORTH WEST PROVINCE, SOUTH AFRICA
}

\author{
Ofodile, N. M. - OgBuewu, I. P. - Mbajiorgu, C. A. - Mokolopi, B. G. \\ University of South Africa, Department of Agriculture and Animal Health, Private Bag, X6, \\ Florida 1710, South Africa \\ *Corresponding author \\ e-mail: kgobebg@unisa.ac.za, gmokolopi@gmail.com; ORCID number: 0000-0002-4849-2636
}

(Received $30^{\text {th }}$ Mar 2021; accepted $10^{\text {th }}$ Jun 2021)

\begin{abstract}
This study was conducted determine the effect of groundnut shell (GNS) on growth rate, body condition score (BCS) and blood mineral status of confined unsexed mixed cattle breed during the winter season in North West province, South Africa. Thirty-five cattle were divided into five groups (T1, T2, T3, T4, and T5) and each group assigned to GNS at 0, 500, 700, 1000 and $1200 \mathrm{gkg}^{-1}$ feed in a completely randomized design. Cattle were fed basal diet (blue buffalo grass; BBG) and results showed that BBG and GNS were moderate in nutrients. Cattle on diet T3 had improved $(\mathrm{p}<0.05)$ live weight, average daily gain and BCS. Animals on diets T3 and T4 had increased $(\mathrm{p}<0.05)$ plasma magnesium and cobalt value than those on diets T1 and T2. Cattle offered diet T3 had increased ( $\mathrm{p}<0.05)$ plasma manganese, iron, carbon, copper and chromium concentrations when compared to those on other four diets. GNS supplementation had no effect $(p>0.05)$ on plasma zinc and selenium. GNS supplementation had quadratic effect $(p<0.05)$ on plasma minerals. It is concluded that GNS contains moderate amount of essential minerals and therefore suitable for use in cattle production during the winter season in North West Province of South Africa.

Keywords: ruminant, performance, blood minerals, agro-byproduct, quadratic function
\end{abstract}

\section{Introduction}

The development of the smallholder cattle industry as an animal protein source for human diets in South Africa has received little attention. Livestock improvement demands efficient use of available feed resources. Factors like agronomic practices, feed processing technologies, and genetic variations have been observed to influence the nutrient (mineral) value of feed raw materials (Khan et al., 2017). Literature is scanty on the mineral status of confined indigenous cattle under a communal grazing system during the dry (winter) season in South Africa or the grass they grazed. Seasonal variability has been reported to affect the minerals status of cattle due to changes in nutrient composition of pasture and their availability (Malau-Aduli et al., 2003; Mokolopi and Beighle, 2006). The long period of dryness usually experienced during the winter season often aggravates mineral deficiency in cattle and this call for detailed studies before a mineral supplement can be recommended. Communal farmers are unwilling to give mineral lick to their cattle for several reasons, of which the most prominent is cost. It is therefore desirable to develop alternative mineral supplement sources in smallholder cattle production especially during the dry season.

Groundnut (Arachis hypogea L) shell one of such novel feed mineral resources that has no direct value in human diet. Shell is a by-product of the groundnut processing industry after removing the seed and comprises about 21-29\% weight of the whole nut (van Doosselaere, 2013; Davis et al., 2016). The shell is high in lignin which calls for 
proper processing before their use in animal ration. Studies have shown that GNS contain $0.50 \%$ crude protein $(\mathrm{CP}), 59.0 \%$ crude fibre $(\mathrm{CF}), 2.50 \%$ ash and $4.43 \%$ carbohydrates (Abdulrazak et al., 2014). According to Atasie et al. (2009), GNS is rich in minerals such as $\mathrm{Na}(42.00 \mathrm{mg} / 100 \mathrm{~g}), \mathrm{K}(705.11 \mathrm{mg} / 100 \mathrm{~g}), \mathrm{Mg}(3.98 .00 \mathrm{mg} / 100 \mathrm{~g}), \mathrm{Ca}$ $(2.28 \mathrm{mg} / 100 \mathrm{~g}), \mathrm{Fe}(6.97 \mathrm{mg} / 100 \mathrm{~g}), \mathrm{Zn}(3.20 \mathrm{mg} / 100 \mathrm{~g})$ and $\mathrm{P}(10.55 \mathrm{mg} / 100 \mathrm{~g})$.

The objective of this study, therefore was to ascertain the nutrient content of GNS and the impact of its supplementation on the growth rate, body condition scores and blood mineral status of unsexed mixed cattle breed fed intensively during the dry (winter) season in North West province, South Africa. The optimal GNS supplementation level that improved LW, ADG, BCS and blood mineral indices will be modelled using quadratic regression model.

\section{Materials and Methods}

\section{Study area}

This study was conducted in Mogosane village of Mafikeng, North West province (Figure 1) during the months of May to July 2017. This town has an above-average rainfall of $300-700 \mathrm{~mm}$ annually and the summer falls between August and March in which the temperatures may range between $22^{\circ} \mathrm{C}$ and $34^{\circ} \mathrm{C}$, while the winter arrives around May to July being dry and cool. The average winter temperature is about $16^{\circ} \mathrm{C}$ which can decline below $2{ }^{\circ} \mathrm{C}$ and can rise to $20^{\circ} \mathrm{C}$.

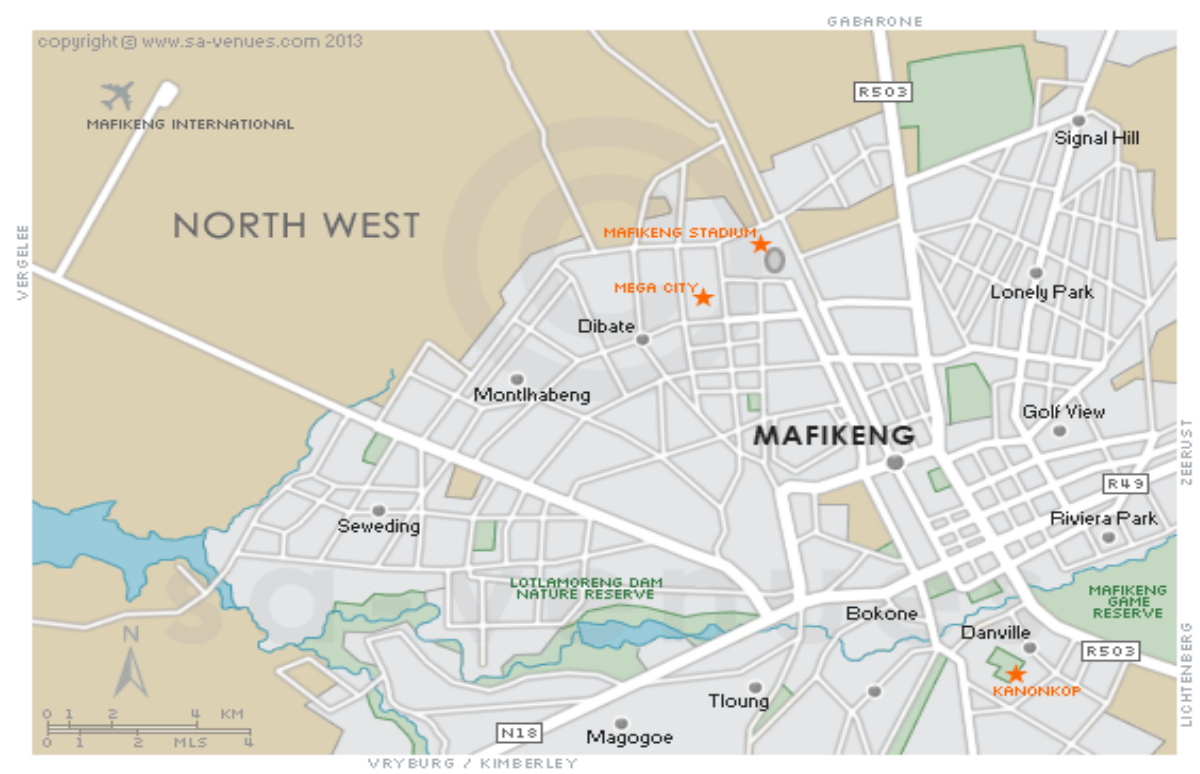

Figure 1. Map of Mafikeng.

Source: https://www.sa-venues.com/maps/northwestprovince/mafikeng.php

\section{Experimental procedure}

Kraals were constructed to have five pens with each pen housing seven (7) cattle. This subdivision aid to prevent the animals from roaming around and missing up in order not to disturb the experimental design. All the biosecurity measures adapted in the current aligned to the guidelines of the Ethics Committee of the University of South Africa and 
the South African Animal Disease Act 35, of 1984. Thirty-five (35) unsexed mixed cattle breed weighing between $237.63 \mathrm{~kg}$ and $278.00 \mathrm{~kg}$ were selected from a herd that is exclusively on communal grazing with no mineral supplement at Mogosane village, Mafikeng and randomly assigned to 5 treatments (T1, T2, T3, T4 and T5) with 7 animals per treatment in a completely randomized design. All the cattle were ear tagged. The animals in control (T1) group received a basal diet supplemented with GNS at $0 \mathrm{~g} / \mathrm{kg}$ feed while those in groups T2, T3, T4 and T5 received basal diet supplemented with GNS at $500,700,1000$ and $1200 \mathrm{gkg}^{-1}$ feed during the feeding periods, respectively. Feed and water were provided ad libitum to the cattle.

\section{Data collection}

Samples of BBG and GNS samples were dried in a forced-air oven at $70^{\circ} \mathrm{C}$ for $48 \mathrm{~h}$ and thereafter, milled and sieved via a 2-mm screen (Retsch Zm100 grinder; Glen Mills Inc., Clifton, NJ) and stored in Fisherbrand sterile sampling bags (Fisher Scientific, Pittsburgh, PA). The milled samples were subjected to proximate analysis to determine their $\mathrm{CP}, \mathrm{CF}$, ether extract (EE), moisture content (MC), ash and nitrogen-free extracts (NFE) following the standard procedures (AOAC, 2002). All the proximate values were analyzed in triplicates and results reported in percentages. Metabolizable energy (ME) was calculated using the prediction equation reported by Pauzenga (1985) as follows: $\mathrm{ME}=37 \times \mathrm{CP} \%+81.8 \times \mathrm{EE} \%+35.5 \times \mathrm{NFE} \%$. Mineral composition of BBG and GNS were determined using inductively coupled plasma optic emission spectroscopy (PerkinElmer, Waltham, MA) as described (Richter et al., 2012). Blood samples were aseptically collected from the jugular vein the four (4) randomly selected animals in each treatment group at the end of the $8^{\text {th }}$ week of the study. As described by Richter et al. (2012), Jugular blood samples for mineral analysis were collected into vacuum tubes containing the anticoagulants potassium EDTA after restraining the animal. Sera were harvested by centrifugation at $1000 \mathrm{rpm}$ for 10 minutes and later transferred into plastic tubes and stored for mineral analysis. Thereafter, the sera were transported to the laboratory for determination of the mineral content. Upon analysis, plasma samples were vortexed, then diluted to a ratio of 1:20 with $1 \%$ nitric acid. Blood samples were analyzed for mineral concentrations using inductively coupled plasma mass spectroscopy (PerkinElmer, Waltham, MA) as described by Pogge et al. (2012). Mean initial live weights (LWs) of the cattle were recorded at the first week of study. Thereafter, mean LW per cattle in each pen were taken on a weekly basis and the total LW were divided by the total number of cattle in the pen to get the mean LW of the cattle. The determined LWs were employed to compute the ADG of the cattle. BCS was determined in a scale of 1 to 5 following the procedures of Wildman et al. (1982). This depends on a visual and tactile appraisal of the body fat reserve in the back and pelvic regions.

\section{Data analysis}

The Statistical Analysis System procedure of SAS 9.4 (SAS Institute, 2010) was used and data collected were analyzed by one-way analysis of variance method to ascertain the influence of GNS supplementation on blood mineral concentrations of South African indigenous cattle. Duncan's Multiple Range (DMR) test was done for multiple means comparison and the differences between means were considered statistically significant at $\mathrm{p}<0.05$. Data generated on the proximate and mineral composition of BBG and GNS were subjected to descriptive statistics [means, standard deviation (SD) and coefficient of variation $(\mathrm{CV})]$ to establish the reference values of the different parameters measured. 
The supplementation related responses in body weight, body condition scores and blood mineral content to GNS supplementation in confined South African indigenous cattle fed intensively during the winter season were modeled using the following quadratic regression model equation:

$$
\mathrm{Y}=\mathrm{a}+\mathrm{b}_{1} \mathrm{x}+\mathrm{b}_{2} \mathrm{X}_{2}
$$

where

$\mathrm{Y}=$ Live weight, body condition score and blood mineral parameters;

$\mathrm{a}=$ the $\mathrm{Y}$-intercept;

$\mathrm{b}=$ coefficient of quadratic optimization equation;

$\mathrm{x}=$ Groundnut shell supplementation levels and $-\mathrm{b}_{1} / 2 \mathrm{~b}_{2}=\mathrm{x}$ value of GNS for optimum response. The quadratic equation was fitted to the experimental data by means of the nonlinear model procedure of SAS (2010). The choice of the quadratic regression model is because it fitted the model and the probability level for significance is $5 \%$.

\section{Results}

\section{Nutrient composition of BBG and GNS}

The mean total ash, $\mathrm{CP}, \mathrm{CF}, \mathrm{EE}, \mathrm{MC}, \mathrm{NFE}$ and ME value of the BBG and GNS are given in Table 1. The SD values across BBG and GNS were low and ranges between $0.03-1.59$ and $0.03-3.74$, respectively. Table 2 showed the macro-mineral profiling of BBG and GNS. BBG yielded $2.12 \mathrm{~g} / 100 \mathrm{~g} \mathrm{Ca}, 0.37 \mathrm{~g} / 100 \mathrm{~g} \mathrm{P}, 0.23 \mathrm{~g} / 100 \mathrm{~g} \mathrm{Mg}$, $0.62 \mathrm{~g} / 100 \mathrm{~g} \mathrm{~K}, 0.26 \mathrm{~g} / 100 \mathrm{~g} \mathrm{~S}, 19.00 \mathrm{~g} / 100 \mathrm{~g} \mathrm{Na}$ and $1.03 \mathrm{~g} / 100 \mathrm{~g} \mathrm{Cl}$, while GNS yielded $0.36 \mathrm{~g} / 100 \mathrm{~g} \mathrm{Ca}, 0.36 \mathrm{~g} / 100 \mathrm{~g} \mathrm{P}, 0.34 \mathrm{~g} / 100 \mathrm{~g} \mathrm{Mg}, 2.23 \mathrm{~g} / 100 \mathrm{~g} \mathrm{~K}, 0.14 \mathrm{~g} / 100 \mathrm{~g} \mathrm{~S}$, $0.05 \mathrm{~g} / 100 \mathrm{~g} \mathrm{Na}$ and $0.18 \mathrm{~g} / 100 \mathrm{~g} \mathrm{Cl}$. SD values across the macro-mineral values for the BBG and GNS were low and ranged between $0.02-1.77$ and $0.01-0.15$, respectively. The mean micro-mineral concentrations of the BBG and GNS were found between 1.85 and $25.01 \mathrm{mg} / 100 \mathrm{~g} \mathrm{Mn}, 2.59$ and $0.32 \mathrm{mg} / 100 \mathrm{~g} \mathrm{Fe}, 1.82$ and $2.67 \mathrm{mg} / 100 \mathrm{~g} \mathrm{Cu}, 6.12$ and $5.04 \mathrm{mg} / 100 \mathrm{~g} \mathrm{Zn}, 60.25$ and $43.87 \mathrm{mg} / 100 \mathrm{~g} \mathrm{C}, 3.18$ and $1.95 \mathrm{mg} / \mathrm{g} \mathrm{Se}, 0.89$ and $0.25 \mathrm{mg} / 100 \mathrm{~g} \mathrm{Cr}, 7.08$ and $0.25 \mathrm{mg} / 100 \mathrm{~g}$ Co and 3.25 and $3.20 \mathrm{mg} / 100 \mathrm{~g} \mathrm{~V}$, respectively (Table 3). Standard deviation values across macro-mineral values were low and ranged $0.12-3.95$ for BBG and $0.02-2.50$ GNS.

Table 1. Proximate analysis (\%) of basal diet and GNS

\begin{tabular}{c|c|c|c|c|c|c|c|c}
\hline \multirow{2}{*}{ Parameters } & \multicolumn{4}{|c|}{ Basal diet } & \multicolumn{4}{c}{ GNS } \\
\cline { 2 - 9 } & Mean & SD & $\begin{array}{c}\text { Min. } \\
\text { Value }\end{array}$ & $\begin{array}{c}\text { Max. } \\
\text { value }\end{array}$ & Mean & SD & $\begin{array}{c}\text { Min. } \\
\text { value }\end{array}$ & $\begin{array}{c}\text { Max. } \\
\text { value }\end{array}$ \\
\hline Total ash & 1.51 & 0.03 & 1.48 & 1.55 & 2.50 & 0.03 & 2.46 & 2.53 \\
CP & 9.09 & 1.03 & 7.87 & 10.4 & 11.67 & 0.59 & 12.01 & 12.16 \\
CF & 18.18 & 1.46 & 16.80 & 20.20 & 49.00 & 3.74 & 45.00 & 54.00 \\
EE & 1.48 & 0.06 & 1.39 & 1.53 & 1.50 & 0.04 & 1.45 & 1.54 \\
Moisture & 15.00 & 0.82 & 14.00 & 16.00 & 8.00 & 0.82 & 7.00 & 9.00 \\
Carbohydrate & 69.74 & 1.59 & 68.53 & 72.00 & 35.33 & 2.06 & 33.00 & 38.00 \\
ME (Kcal/kg) * & 2839.92 & 8.02 & 2829.89 & 2849.52 & 1714.21 & 21.17 & 1696.55 & 1743.98 \\
\hline
\end{tabular}

*Calculated 
Table 2. Macro-mineral value of basal diet and GNS measured in dry weight ( $\mathrm{g} / 100 \mathrm{~g})$

\begin{tabular}{c|cccc|cccc}
\hline \multirow{2}{*}{ Minerals } & \multicolumn{4}{|c|}{ Basal diet } & \multicolumn{4}{c}{ GNS } \\
\cline { 2 - 9 } & Mean & SD & $\begin{array}{c}\text { Min. } \\
\text { Value }\end{array}$ & $\begin{array}{c}\text { Max. } \\
\text { value }\end{array}$ & Mean & SD & $\begin{array}{c}\text { Min. } \\
\text { Value }\end{array}$ & $\begin{array}{c}\text { Max. } \\
\text { value }\end{array}$ \\
\hline $\mathrm{Ca}$ & 2.12 & 0.30 & 1.76 & 2.50 & 0.36 & 0.06 & 0.31 & 0.42 \\
$\mathrm{P}$ & 0.37 & 0.02 & 0.35 & 0.40 & 0.36 & 0.03 & 0.34 & 0.40 \\
$\mathrm{Mg}$ & 0.23 & 0.02 & 0.21 & 0.25 & 0.34 & 0.05 & 0.29 & 0.40 \\
$\mathrm{~K}$ & 0.62 & 0.02 & 0.60 & 0.65 & 2.23 & 0.15 & 2.02 & 2.43 \\
$\mathrm{~S}$ & 0.26 & 0.03 & 0.24 & 0.30 & 0.14 & 0.01 & 0.13 & 0.15 \\
$\mathrm{Na}$ & 19.00 & 1.77 & 16.5 & 20.42 & 0.05 & 0.02 & 0.03 & 0.07 \\
$\mathrm{Cl}$ & 1.03 & 0.04 & 1.00 & 1.08 & 0.18 & 0.02 & 0.16 & 0.20 \\
\hline
\end{tabular}

Table 3. Micro-mineral value of basal diet and GNS measured in dry weight ( $\mathrm{mg} / 100 \mathrm{~g})$

\begin{tabular}{c|cccc|cccc}
\hline \multirow{2}{*}{ Minerals } & \multicolumn{4}{|c|}{ Basal diet } & \multicolumn{4}{c}{ GNS } \\
\cline { 2 - 9 } & Mean & SD & $\begin{array}{c}\text { Min. } \\
\text { value }\end{array}$ & $\begin{array}{c}\text { Max. } \\
\text { value }\end{array}$ & Mean & SD & $\begin{array}{c}\text { Min. } \\
\text { value }\end{array}$ & $\begin{array}{c}\text { Max. } \\
\text { value }\end{array}$ \\
\hline $\mathrm{Mn}$ & 1.85 & 0.17 & 1.62 & 2.01 & 25.01 & 1.45 & 23.6 & 27.01 \\
$\mathrm{Fe}$ & 2.59 & 0.38 & 2.19 & 3.10 & 0.32 & 0.11 & 0.20 & 0.46 \\
$\mathrm{Cu}$ & 1.82 & 0.12 & 1.68 & 1.98 & 2.67 & 0.28 & 2.40 & 3.05 \\
$\mathrm{Zn}$ & 6.12 & 0.12 & 5.98 & 6.40 & 5.04 & 0.86 & 4.16 & 6.20 \\
$\mathrm{C}$ & 60.25 & 3.95 & 58.85 & 67.79 & 43.87 & 2.50 & 40.40 & 46.21 \\
$\mathrm{Se}$ & 3.18 & 0.21 & 2.99 & 3.47 & 1.95 & 0.20 & 1.68 & 2.14 \\
$\mathrm{Cr}$ & 0.89 & 0.18 & 0.65 & 1.08 & 0.25 & 0.02 & 0.22 & 0.27 \\
$\mathrm{Co}$ & 7.08 & 1.33 & 5.32 & 8.55 & 0.25 & 0.04 & 0.20 & 0.30 \\
$\mathrm{~V}$ & 3.25 & 0.56 & 2.71 & 4.02 & 3.20 & 0.28 & 3.00 & 3.60 \\
\hline
\end{tabular}

\section{Growth rate, condition score and blood mineral characteristics of cattle}

Data on the effect of GNS supplementation on LW, ADG, body condition score (BCS) and plasma macro-mineral concentrations of indigenous cattle managed intensively during the winter season in North West province of South Africa are shown in Figures 2, 3,4 and Table 4. Cattle on diet T3 had improved $(\mathrm{p}<0.05)$ growth rate and body condition score when compared to those on other 4 diets starting from the $4^{\text {th }}$ week of the study. Mean $\mathrm{Ca}, \mathrm{Mg}, \mathrm{P}, \mathrm{K}, \mathrm{Na}, \mathrm{Cl}$ and $\mathrm{S}$ value were $3.256 \mathrm{mg} \%, 0.609 \mathrm{mg} \%, 0.308 \mathrm{mg} \%$, $4.799 \mathrm{mg} \%, 15.599 \mathrm{mg} \%, 6919.8 \mathrm{mg} \%$ and $48.177 \mathrm{mg} \%$, respectively, while the CV was ranged from 11.20 to $58.50 \%$. Cattle fed diet T2, T3, T4 and T5 had higher $(\mathrm{p}<0.05)$ plasma Ca when compared with cattle on diet T1. Cattle on diets T3 and T4 had significantly $(\mathrm{p}<0.05)$ increased plasma $\mathrm{Mg}$ concentration when compared to those on $\mathrm{T} 1$ and T2 diets. However, cattle on diets T1 and T2 had similar ( $>>0.05$ ) plasma Mg. Plasma $\mathrm{P}$ and $\mathrm{K}$ concentrations of cattle fed diet $\mathrm{T} 1$ was significantly $(\mathrm{p}<0.05)$ lower than those fed diets T3, T4 and T5, but had similar value with those fed diet T2. Cattle on diets T3, T4 and T5 had the highest plasma Na values which differed significantly from those on diet T1. Our result also revealed that cattle on diets $\mathrm{T} 1$ and $\mathrm{T} 2$ had similar $(\mathrm{p}<0.05)$ plasma $\mathrm{Na}$ which differed significantly from those on diets T3 and T4. Furthermore, our results showed statistical $(\mathrm{p}<0.05)$ effect of GNS supplementation on plasma $\mathrm{Cl}$ and $\mathrm{S}$ among the dietary groups. 




Figure 2. Live weight of unsexed mixed cattle breed on fed basal diet supplemented with GNS. ${ }_{a, b, c}$ Bars sharing different letters are significant at $p<0.05$

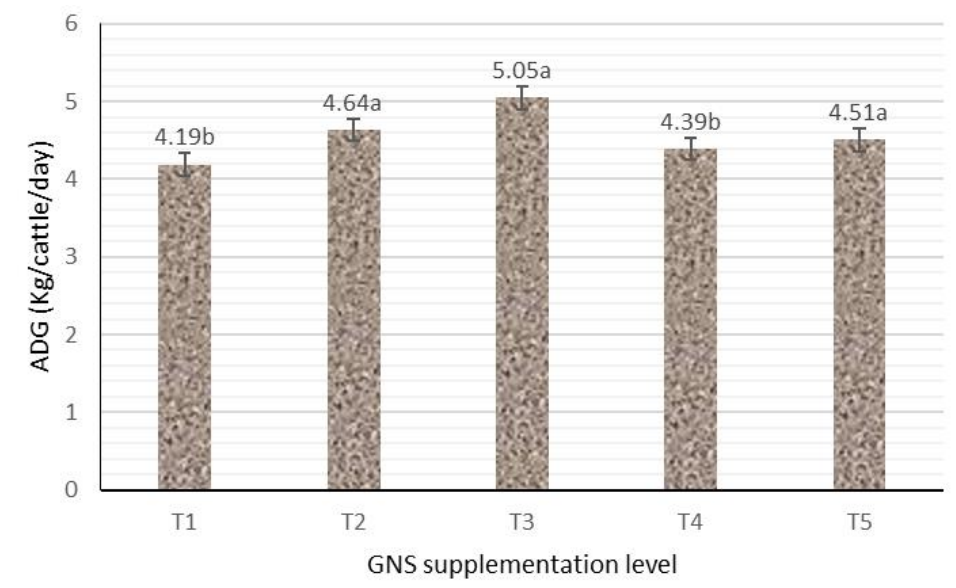

Figure 3. ADG value of unsexed mixed cattle breed fed basal diet supplemented with GNS.

${ }^{a, b}$ Bars sharing different letters are significant at $p<0.05$

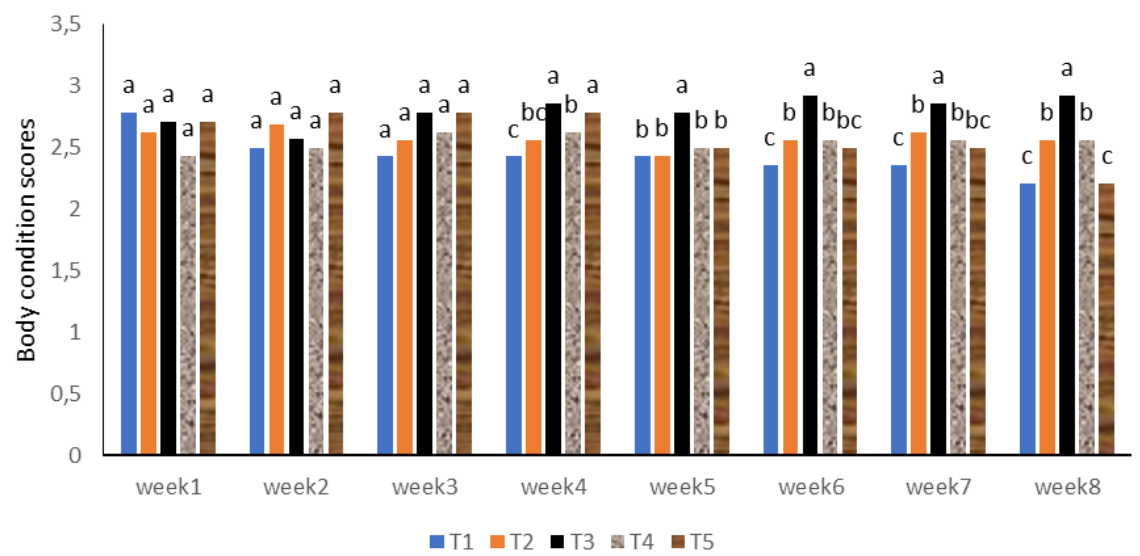

Figure 4. Body condition scores of indigenous cattle on fed basal diet supplemented with GNS. ${ }^{a, b, c}$ Bars sharing different letters are significant at $p<0.05$ 
Table 4. Blood macro-mineral content (mg\%) of cattle fed diets supplemented with GNS

\begin{tabular}{|c|c|c|c|c|c|c|c|c|c|}
\hline \multirow{2}{*}{ Variables } & \multicolumn{5}{|c|}{ GNS supplementation levels (g/kg feed) } & \multirow{2}{*}{ Mean } & \multirow{2}{*}{ SEM } & \multirow{2}{*}{ p-value } & \multirow{2}{*}{$\% \mathrm{CV}$} \\
\hline & T1 & $\mathbf{T 2}$ & T3 & T4 & T5 & & & & \\
\hline $\mathrm{Ca}$ & $2.479^{\mathrm{a}}$ & $2.930^{c}$ & $4.383^{\mathrm{b}}$ & $3.736^{\mathrm{b}}$ & $2.751^{\mathrm{c}}$ & 3.256 & 0.223 & 0.0001 & 24.10 \\
\hline $\mathrm{Mg}$ & $0.206^{\mathrm{d}}$ & $0.270^{\text {cd }}$ & $0.433^{\mathrm{a}}$ & $0.354^{\mathrm{b}}$ & $0.279^{c}$ & 0.609 & 0.022 & 0.0001 & 31.70 \\
\hline $\mathrm{P}$ & $0.231^{\mathrm{c}}$ & $0.314 \mathrm{c}$ & $1.116^{\mathrm{a}}$ & $0.756^{\mathrm{b}}$ & $0.627^{\mathrm{b}}$ & 0.308 & 0.050 & 0.0001 & 58.50 \\
\hline K & $3.614^{\mathrm{d}}$ & $3.900^{\text {cd }}$ & $6.186^{\mathrm{a}}$ & $5.514^{\mathrm{ab}}$ & $4.785^{\mathrm{bc}}$ & 4.799 & 0.373 & 0.0001 & 22.40 \\
\hline $\mathrm{Na}$ & $12.414^{\mathrm{a}}$ & $13.759^{\mathrm{ab}}$ & $19.880^{\mathrm{c}}$ & $17.326^{\mathrm{c}}$ & $14.614^{\mathrm{bc}}$ & 15.599 & 1.247 & 0.0015 & 19.40 \\
\hline $\mathrm{Cl}$ & $6359.4^{c}$ & $6573.6^{\mathrm{bc}}$ & $8106.0^{\mathrm{a}}$ & $7308.4^{\mathrm{ab}}$ & $6251.6^{\mathrm{c}}$ & 6919.8 & 522.3 & 0.0390 & 11.20 \\
\hline$S$ & $44.847^{\mathrm{bc}}$ & $48.443^{\mathrm{b}}$ & $58.693^{\mathrm{a}}$ & $47.443^{\mathrm{b}}$ & $41.457^{\mathrm{c}}$ & 48.177 & 0.531 & 0.0220 & 14.30 \\
\hline
\end{tabular}

a,b,c,d Means within rows sharing different letters are significant at $\mathrm{p}<0.05$. SEM: Standard error of the mean; CV: Coefficient of variation

Results of the effect of GNS supplementation on plasma micro-mineral concentrations of indigenous cattle managed intensively during the winter season are presented in Table 5. Mean $\mathrm{Mn}, \mathrm{Fe}, \mathrm{Cu}, \mathrm{Zn}, \mathrm{C}, \mathrm{Se}, \mathrm{Cr}$, Co and $\mathrm{V}$ concentration were $0.0456 \mathrm{mg} \%$, $0.1206 \mathrm{mg} \%, 0.0216 \mathrm{mg} \%, 0.0014 \mathrm{mg} \%, 756.68 \mathrm{mg} \%, 0.0022 \mathrm{mg} \%, 0.0218 \mathrm{mg} \%$, $0.2238 \mathrm{mg} \%$ and $0.074 \mathrm{mg} \%$, respectively while the coefficient of variations (CV) was ranged from 11.20 to $58.50 \%$. Indigenous cattle offered diet T3 had higher $(\mathrm{p}<0.05)$ plasma $\mathrm{Mn}, \mathrm{Fe}$ and $\mathrm{C}$ value than those on diets T1, T2, T4 and T5. However, cattle fed diets T1, T2, T4 and T5 had comparable (p>0.05) plasma Mn, Fe and C value. Cattle on diet $\mathrm{T} 3$ had higher $(\mathrm{p}<0.05)$ plasma $\mathrm{Cu}$ concentration than those on the other 4 diets. However, cattle offered diets $\mathrm{T} 1 \mathrm{had}$ the lowest $(\mathrm{p}<0.05)$ plasma $\mathrm{Cu}$ value than those on diets T3, T4 and T5, but similar to those on diet T2. Cattle fed diets T1, T2, T3, T4 and T5 had similar $(\mathrm{p}>0.05)$ plasma $\mathrm{Zn}$ and Se concentration. Higher $(\mathrm{p}<0.05)$ plasma Co was recorded in cattle fed diet T3 followed by those fed diets T4 and T5 when compared with those on diet T1 and T2. However, cattle fed diets T1 and T2 had similar ( $\mathrm{p}>0.05)$ plasma Co level. Animals offered diet T3 had increased $(\mathrm{p}<0.05)$ plasma $\mathrm{Cr}$ than those fed diet T5 but similar ( $>>0.05)$ to those on diets T1, T2 and T4. Cattle fed diet T5 had significantly $(\mathrm{p}<0.05)$ reduced plasma $\mathrm{V}$ when compared to those on diets $\mathrm{T} 3$ and $\mathrm{T} 4$. However, cattle on diets T1, T2 and T4 had similar ( $>>0.05$ ) plasma V.

Table 5. Blood micro-mineral contents (mg\%) of cattle fed diets supplemented with GNS

\begin{tabular}{|c|c|c|c|c|c|c|c|c|c|}
\hline \multirow{2}{*}{ Variables } & \multicolumn{5}{|c|}{ GNS supplementation levels (g/kg feed) } & \multirow{2}{*}{ Mean } & \multirow{2}{*}{ SEM } & \multirow{2}{*}{ p-value } & \multirow{2}{*}{$\% \mathrm{CV}$} \\
\hline & T1 & $\mathbf{T 2}$ & T3 & T4 & T5 & & & & \\
\hline $\mathrm{Mn}$ & $0.031^{\mathrm{b}}$ & $0.032^{\mathrm{b}}$ & $0.100^{\mathrm{a}}$ & $0.035^{\mathrm{b}}$ & $0.030^{\mathrm{b}}$ & 0.0456 & 0.009 & 0.0001 & 66.90 \\
\hline $\mathrm{Fe}$ & $0.103^{\mathrm{b}}$ & $0.111^{\mathrm{b}}$ & $0.176^{\mathrm{a}}$ & $0.114^{\mathrm{b}}$ & $0.099^{\mathrm{b}}$ & 0.1206 & 0.008 & 0.0001 & 26.10 \\
\hline $\mathrm{Cu}$ & $0.008^{\mathrm{d}}$ & $0.010^{\mathrm{d}}$ & $0.042^{\mathrm{a}}$ & $0.028^{\mathrm{b}}$ & $0.020^{\mathrm{c}}$ & 0.0216 & 0.001 & 0.0001 & 64.60 \\
\hline $\mathrm{Zn}$ & 0.001 & 0.001 & $0.003^{\mathrm{a}}$ & 0.001 & 0.001 & 0.0014 & 0.401 & 0.5228 & 21.90 \\
\hline $\mathrm{C}$ & $642.70^{\mathrm{b}}$ & $702.5^{b}$ & $990.1^{\mathrm{a}}$ & $805.4^{\mathrm{b}}$ & $642.7^{\mathrm{b}}$ & 756.68 & 54.56 & 0.0003 & 19.30 \\
\hline $\mathrm{Se}$ & $0.002^{\mathrm{a}}$ & $0.002^{\mathrm{a}}$ & $0.003^{\mathrm{a}}$ & $0.002^{\mathrm{a}}$ & $0.002^{\mathrm{a}}$ & 0.0022 & 0.001 & 0.5238 & 20.30 \\
\hline $\mathrm{Cr}$ & $0.021^{\mathrm{ab}}$ & $0.022^{\mathrm{ab}}$ & $0.026^{\mathrm{a}}$ & $0.022^{\mathrm{ab}}$ & $0.018^{b}$ & 0.0218 & 0.002 & 0.0001 & 13.10 \\
\hline Co & $0.026^{\mathrm{d}}$ & $0.027^{\mathrm{cd}}$ & $0.433^{\mathrm{a}}$ & $0.354^{\mathrm{b}}$ & $0.279^{c}$ & 0.2238 & 0.022 & 0.0001 & 19.80 \\
\hline $\mathrm{V}$ & $0.072^{\mathrm{bc}}$ & $0.074^{\mathrm{abc}}$ & $0.088^{\mathrm{a}}$ & $0.077^{\mathrm{ab}}$ & $0.059^{c}$ & 0.0740 & 0.005 & 0.0093 & 13.90 \\
\hline
\end{tabular}

${ }_{a, b, c, d}$ Means within rows sharing different letters are significant at $\mathrm{p}<0.05$ 


\section{Optimization function}

Results of the effect of GNS supplementation on optimal plasma GR, BCS, Ca, P, Mg, $\mathrm{K}, \mathrm{Na}, \mathrm{Cl}$ and $\mathrm{S}$ level in cattle managed intensively during the winter season in North West province of South Africa are shown in Table 6 and Figures 5 to 14. GNS was noticed to have a significant quadratic effect on optimal LW, ADG, BCS and plasma K level with a quadratic value of $199.8+43.968 \mathrm{GNS}-6.9597 \mathrm{GNS}^{2}, \mathrm{r}^{2}=0.5519$; $3.574+0.7804 \mathrm{GNS}-0.1236 \mathrm{GNS}^{2}, \mathrm{r}^{2}=0.5522 ; 2.1714+0.3166 \mathrm{GNS}-0.0482 \mathrm{GNS}^{2}$, $\mathrm{r}^{2}=0.5662$ and $1.119+2.5333 \mathrm{GNS}-0.3563 \mathrm{GNS}^{2}, \mathrm{r}^{2}=0.7191$ with the optimum GNS supplementation level being 315.88, 328.42 and $355.0 \mathrm{~g} / \mathrm{kg}$ feed, respectively. Similar quadratic effect were observed for plasma Ca $\left(0.3648+2.2659 \mathrm{GNS}-0.3551 \mathrm{GNS}^{2}\right.$, $\left.\mathrm{r}^{2}=0.7901\right)$, plasma $\mathrm{P}\left(-0.5544+0.8031 \mathrm{GNS}-0.1133 \mathrm{GNS}^{2}, \mathrm{r}^{2}=0.6523\right)$, plasma $\mathrm{Mg}\left(-0.0206+0.2459 \mathrm{GNS}-0.0371 \mathrm{GNS}^{2}, \mathrm{r}^{2}=0.8086\right)$, plasma Na $(4.814+7.992 \mathrm{GNS}$ $\left.-1.1992 \mathrm{GNS}^{2}, \mathrm{r}^{2}=0.7395\right)$, plasma Cl $\left(4328+2139.9 \mathrm{GNS}-348 \mathrm{GNS}^{2}, \mathrm{r}^{2}=0.7063\right)$ and plasma $\mathrm{S}\left(30.179+16.649 \mathrm{GNS}-2.9046 \mathrm{GNS}^{2}, \mathrm{r}^{2}=0.7415\right)$ level with optimum GNS supplementation being 319.05, 354.41, 331.40, 333.22, 307.46 and $286.60 \mathrm{~g} / \mathrm{kg}$ feed, respectively (Table 4.6). Coefficient of determination $\left(\mathrm{r}^{2}\right)$ was ranged from $55.19 \%$ to $80.86 \%$. However, the level of GNS needed to optimize plasma K was higher than the level needed for optimizing the growth rate (GR), body condition score (BCS), Ca, P, $\mathrm{Mg}, \mathrm{Na}, \mathrm{Cl}$ and $\mathrm{S}$.

Table 6. Optimal GR, BCS and plasma micro-mineral level of cattle on GNS supplementation

\begin{tabular}{c|c|c|c|c|c}
\hline Variables & Optimization Equation & $\mathbf{r}^{2}$ & Optimal X - level & Optimal Y- level & P-value \\
\hline $\mathrm{LW}$ & $\mathrm{Y}=199.8+43.968 \mathrm{x}-6.9597 \mathrm{x}^{2}$ & 0.5519 & 315.88 & 269.24 & $<0.0001$ \\
$\mathrm{ADG}$ & $\mathrm{Y}=3.574+0.7804 \mathrm{x}-0.1236 \mathrm{x}^{2}$ & 0.5522 & 315.70 & 4.8058 & 0.0001 \\
$\mathrm{BCS}$ & $\mathrm{Y}=2.1714+0.3166 \mathrm{x}-0.0482 \mathrm{x}^{2}$ & 0.5662 & 328.42 & 2.6913 & 0.0002 \\
$\mathrm{Ca}$ & $\mathrm{Y}=0.3648+2.2659 \mathrm{x}-0.3551 \mathrm{x}^{2}$ & 0.7901 & 319.05 & 3.9795 & 0.0001 \\
$\mathrm{P}$ & $\mathrm{Y}=-0.5544+0.8031 \mathrm{x}-0.1133 \mathrm{x}^{2}$ & 0.6523 & 354.41 & 1.4231 & 0.0001 \\
$\mathrm{Mg}$ & $\mathrm{Y}=-0.0206+0.2459 \mathrm{x}-0.0371 \mathrm{x}^{2}$ & 0.8086 & 331.40 & 0.3869 & 0.0001 \\
$\mathrm{~K}$ & $\mathrm{Y}=1.119+2.5333 \mathrm{x}-0.3563 \mathrm{x}^{2}$ & 0.7191 & 355.0 & 5.6220 & 0.0001 \\
$\mathrm{Na}$ & $\mathrm{Y}=4.814+7.992 \mathrm{x}-1.1992 \mathrm{x}^{2}$ & 0.7395 & 333.22 & 18.1296 & 0.0015 \\
$\mathrm{Cl}$ & $\mathrm{Y}=4328+2139.9 \mathrm{x}-348 \mathrm{x}^{2}$ & 0.7063 & 307.46 & 7617.64 & 0.0390 \\
$\mathrm{~S}$ & $\mathrm{Y}=30.179+16.649 \mathrm{x}-2.9046 \mathrm{x}^{2}$ & 0.7415 & 286.60 & 54.047 & 0.0220 \\
\hline
\end{tabular}

$\mathrm{r}^{2}$ : Coefficient of determination; p: probability; GR: growth rate; BCS: body condition score; LW: live weight; ADG: average daily gain; GNS: groundnut shell

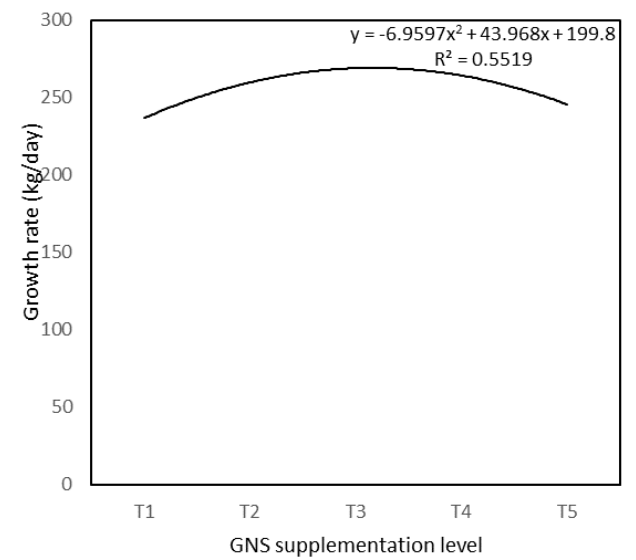

Figure 5. Estimation curve of the optimal live weight of unsexed mixed cattle breed fed basal diet supplemented with GNS 




Figure 6. Estimation curve of the optimal ADG of unsexed mixed cattle breed fed basal diet supplemented with GNS

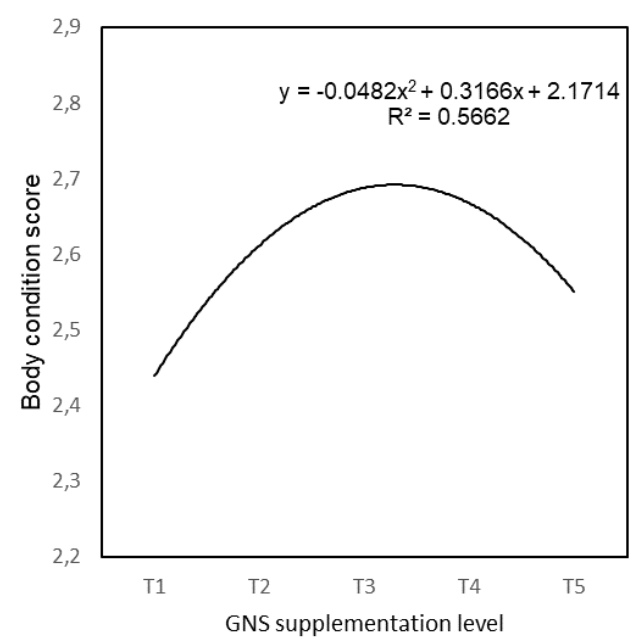

Figure 7. Estimation curve of the optimal BCS of unsexed mixed cattle breed fed basal diet supplemented with GNS

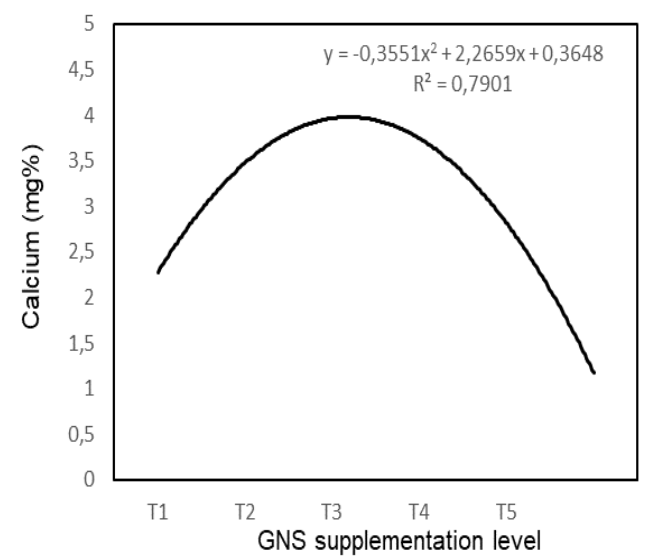

Figure 8. Estimation curve of the optimal plasma Ca of unsexed mixed cattle breed fed basal diet supplemented with GNS 


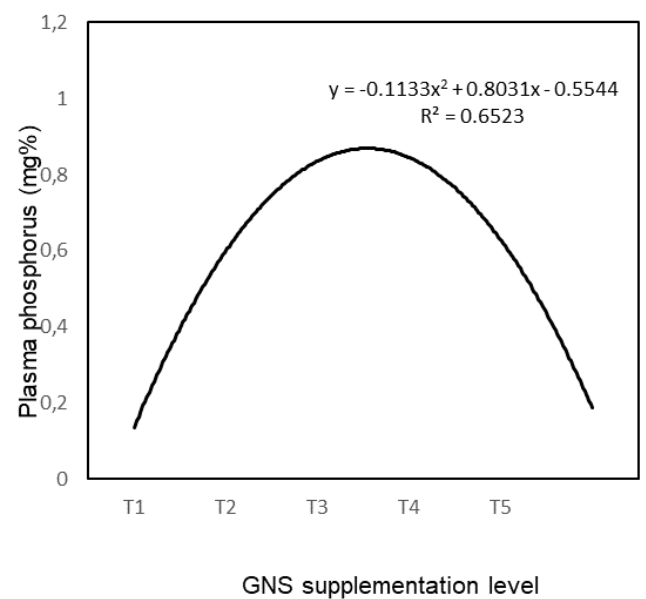

Figure 9. Estimation curve of the optimal plasma $P$ of unsexed mixed cattle breed fed basal diet supplemented with GNS

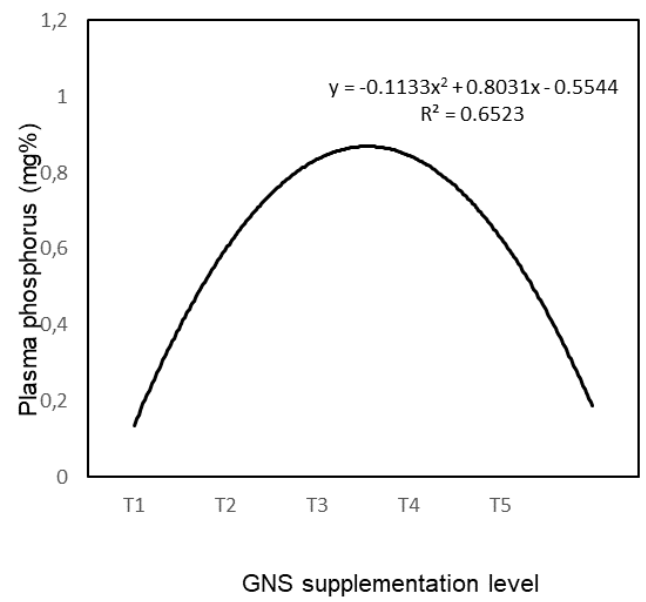

Figure 10. Estimation curve of the optimal plasma Mg of unsexed mixed cattle breed fed basal diet supplemented with GNS

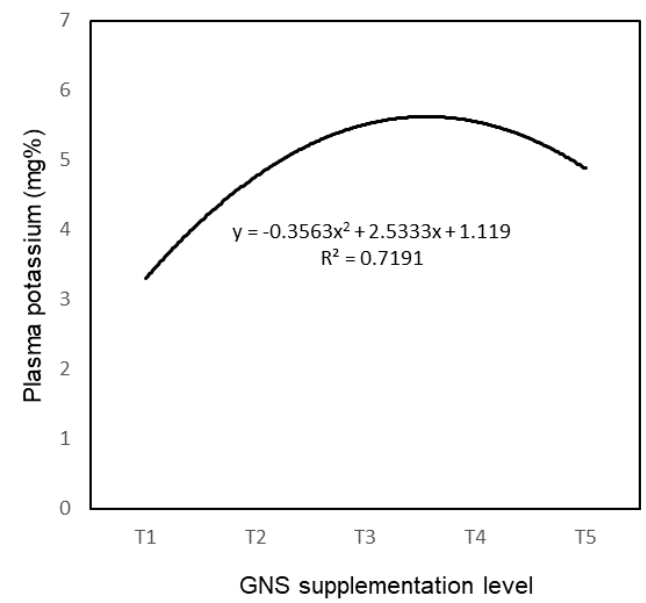

Figure 11. Estimation curve of the optimal plasma K of unsexed mixed cattle breed fed basal diet supplemented with GNS 


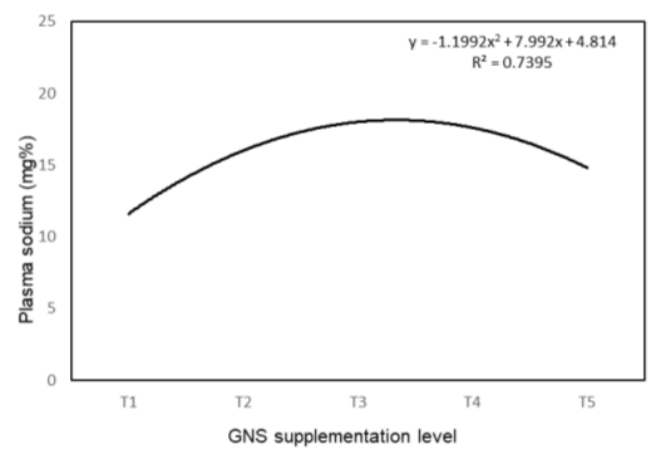

Figure 12. Estimation curve of the optimal plasma Na of unsexed mixed cattle breed fed basal diet supplemented with GNS

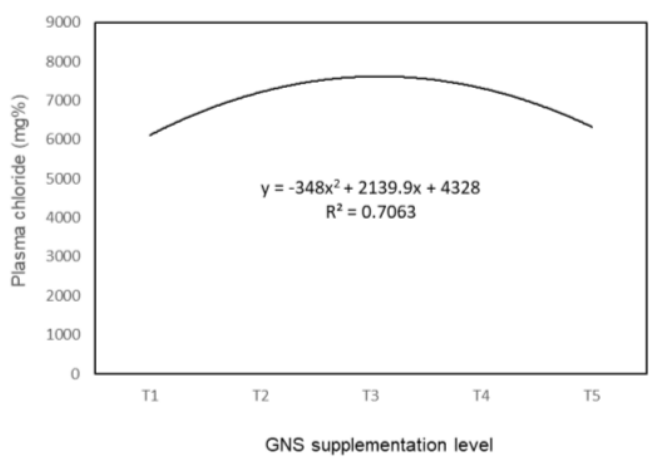

Figure 13. Estimation curve of the optimal plasma Cl of unsexed mixed cattle breed fed basal diet supplemented with GNS

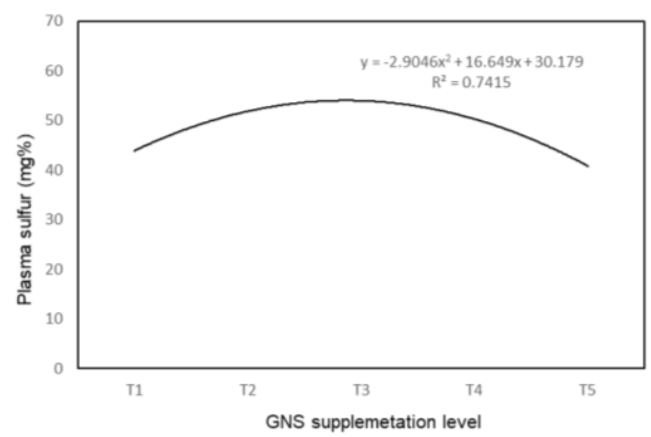

Figure 14. Estimation curve of the optimal plasma $S$ of unsexed mixed cattle breed fed basal diet supplemented with GNS

Results of the effect of GNS supplementation on optimal plasma $\mathrm{Mn}, \mathrm{Fe}, \mathrm{Cu}, \mathrm{Zn}, \mathrm{C}$, $\mathrm{Se}, \mathrm{Cr}$, Co and $\mathrm{V}$ level in cattle managed intensively during the winter season in North West province of South Africa are shown in Table 7 and Figures 15 to 23. GNS was observed to have a quadratic effect $(\mathrm{p}<0.05)$ on optimal plasma Co level with a quadratic value of $-0.3446+0.3563 \mathrm{GNS}-0.0455 \mathrm{GNS}^{2}, \mathrm{r}^{2}=0.6946$ with the optimum GNS supplementation level being $3.9154 \mathrm{~g} / \mathrm{kg}$ feed. Similar quadratic effect were observed for plasma Mn $\left(-0.0272+0.0622 \mathrm{GNS}-0.0104 \mathrm{GNS}^{2}, \mathrm{r}^{2}=0.4045\right)$, plasma Fe $(-0.0356+$ 0.0736 GNS $\left.-0.0124 \mathrm{GNS}^{2}, \mathrm{r}^{2}=0.5376\right)$, plasma $\mathrm{Cu}(-0.024+0.0325$ GNS -0.0047 
$\left.\mathrm{GNS}^{2}, \mathrm{r}^{2}=0.6257\right)$, plasma carbon $\left(267.16+403.42 \mathrm{GNS}-65.521 \mathrm{GNS}^{2}, \mathrm{r}^{2}=0.7130\right)$, plasma $\mathrm{Cr}\left(0.0146+0.0071 \mathrm{GNS}-0.0013 \mathrm{GNS}^{2}, \mathrm{r}^{2}=0.8153\right)$ and plasma V $(0.0484+$ 0.0256 GNS $\left.-0.0046 \mathrm{GNS}^{2}, \mathrm{r}^{2}=0.8172\right)$ level with optimum GNS supplementation being 299.04, 296.77, 345.74, 307.86, 273.08 and $078.26 \mathrm{~g} / \mathrm{kg}$ feed, respectively (Table 7). Coefficient of determination $\left(\mathrm{r}^{2}\right)$ was ranged from 35.71 to 80.72 . However, the level of GNS needed to optimize plasma potassium was higher than the level needed for optimizing the $\mathrm{Ca}, \mathrm{P}, \mathrm{Mg}, \mathrm{Na}, \mathrm{Cl}$ and $\mathrm{S}$. GNS supplementation had no quadratic effect ( $p>0.05)$ on optimal plasma Zn $\left(-0.0012+0.0022\right.$ GNS $\left.-0.0004 \mathrm{GNS}^{2}, \mathrm{r}^{2}=0.5893\right)$ and plasma Se $\left(0.0012+0.0009 \mathrm{GNS}-0.0001 \mathrm{GNS}^{2}, \mathrm{r}^{2}=0.3571\right)$.

Table 7. Optimal plasma micromineral level ( $\mathrm{mg} \%)$ value of cattle to GNS supplementation

\begin{tabular}{c|c|c|c|c|c}
\hline Variable & Equation & $\mathbf{r}^{2}$ & $\begin{array}{c}\text { Optimal } \\
\text { X level }\end{array}$ & $\begin{array}{c}\text { Optimal } \\
\text { Y level }\end{array}$ & p-value \\
\hline $\mathrm{Mn}$ & $\mathrm{Y}=-0.0272+0.0622 \mathrm{x}-0.0104 \mathrm{x}^{2}$ & 0.4045 & 299.04 & 0.0658 & 0.0001 \\
$\mathrm{Fe}$ & $\mathrm{Y}=-0.0356+0.0736 \mathrm{x}-0.0124 \mathrm{x}^{2}$ & 0.5376 & 296.77 & 0.1092 & 0.0001 \\
$\mathrm{Cu}$ & $\mathrm{Y}=-0.024+0.0325 \mathrm{x}-0.0047 \mathrm{x}^{2}$ & 0.6257 & 345.74 & 0.0322 & 0.0001 \\
$\mathrm{Zn}$ & $\mathrm{Y}=-0.0012+0.0022 \mathrm{x}-0.0004 \mathrm{x}^{2}$ & 0.5893 & 275.00 & 0.0018 & 0.5228 \\
$\mathrm{C}$ & $\mathrm{Y}=267.16+403.42 \mathrm{x}-65.521 \mathrm{x}^{2}$ & 0.7130 & 307.86 & 888.14 & 0.0003 \\
$\mathrm{Se}$ & $\mathrm{Y}=0.0012+0.0009 \mathrm{x}-0.0001 \mathrm{x}^{2}$ & 0.3571 & 450.00 & 0.0008 & 0.5238 \\
$\mathrm{Cr}$ & $\mathrm{Y}=0.0146+0.0071 \mathrm{x}-0.0013 \mathrm{x}^{2}$ & 0.8153 & 273.08 & 0.0243 & 0.0001 \\
$\mathrm{Co}$ & $\mathrm{Y}=-0.3446+0.3563 \mathrm{x}-0.0455 \mathrm{x}^{2}$ & 0.6946 & 391.54 & 0.3529 & 0.0001 \\
$\mathrm{~V}$ & $\mathrm{Y}=0.0484+0.0256 \mathrm{x}-0.0046 \mathrm{x}^{2}$ & 0.8172 & 78.26 & 0.0840 & 0.0093 \\
\hline
\end{tabular}

$r^{2}$ : Coefficient of determination; $p$ : probability

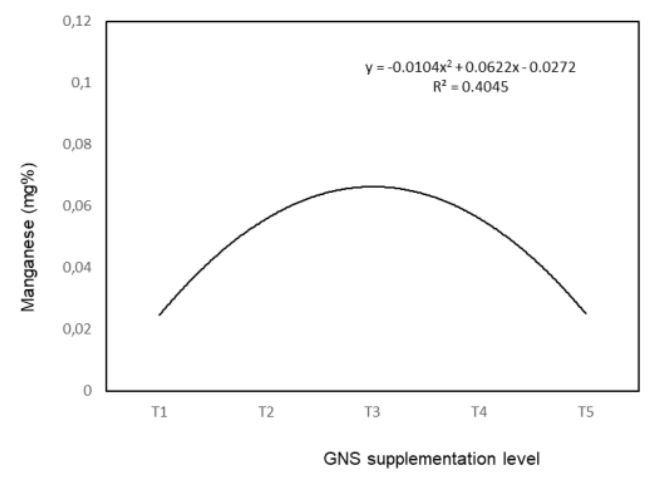

Figure 15. Estimation curve of the optimal plasma Mn of unsexed mixed cattle breed fed basal diet supplemented with GNS

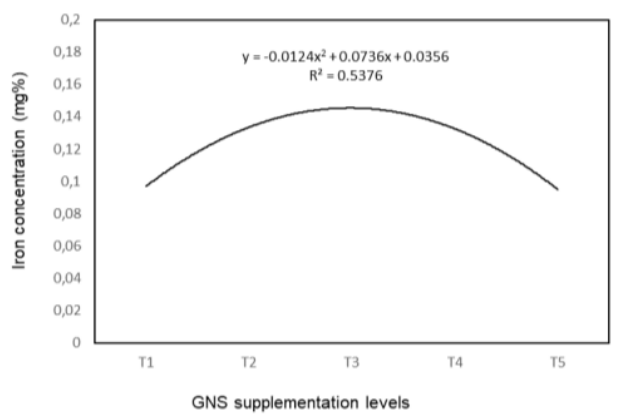

Figure 16. Estimation curve of the optimal plasma Fe of unsexed mixed cattle breed fed basal diet supplemented with GNS 




Figure 17. Estimation curve of the optimal plasma $\mathrm{Cu}$ of unsexed mixed cattle breed fed basal diet supplemented with GNS

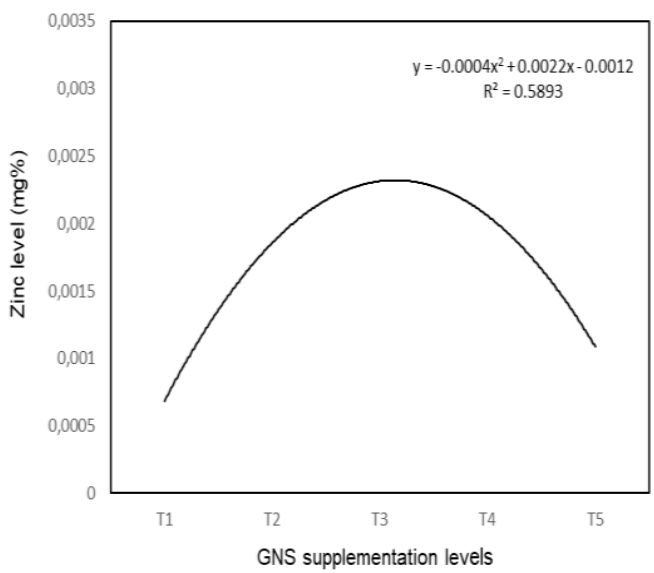

Figure 18. Estimation curve of the optimal plasma Zn of unsexed mixed cattle breed fed basal diet supplemented with GNS

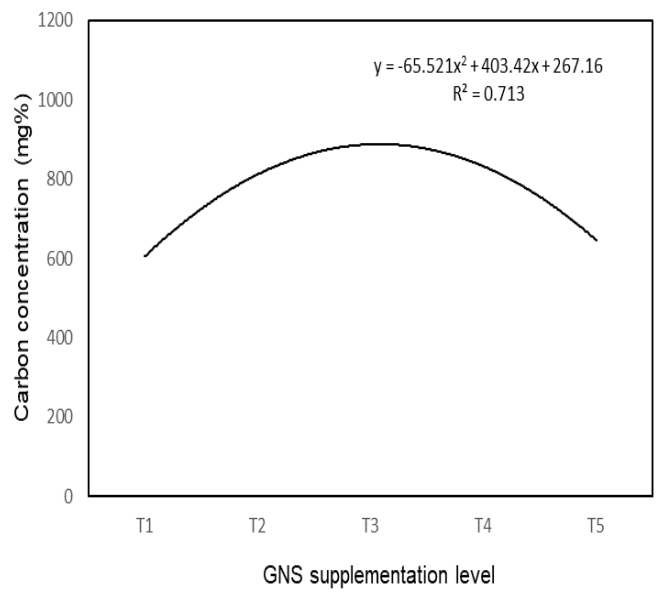

Figure 19. Estimation curve of the optimal plasma $C$ of unsexed mixed cattle breed fed basal diet supplemented with GNS 


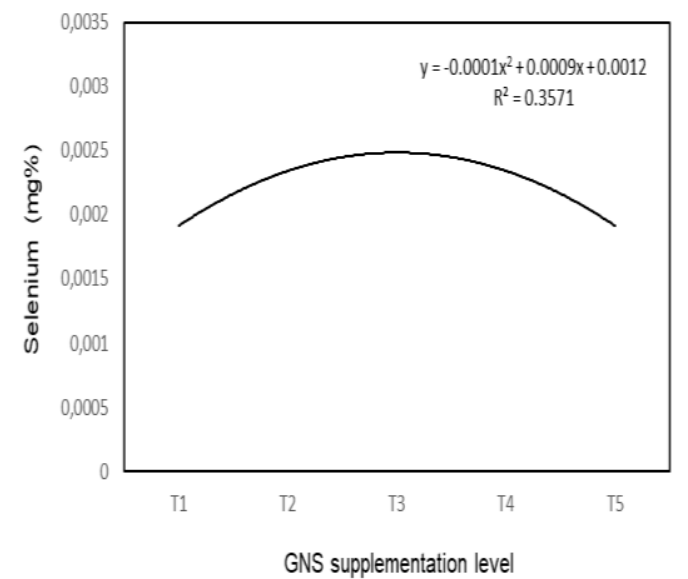

Figure 20. Estimation curve of the optimal plasma Se of unsexed mixed cattle breed fed basal diet supplemented with GNS

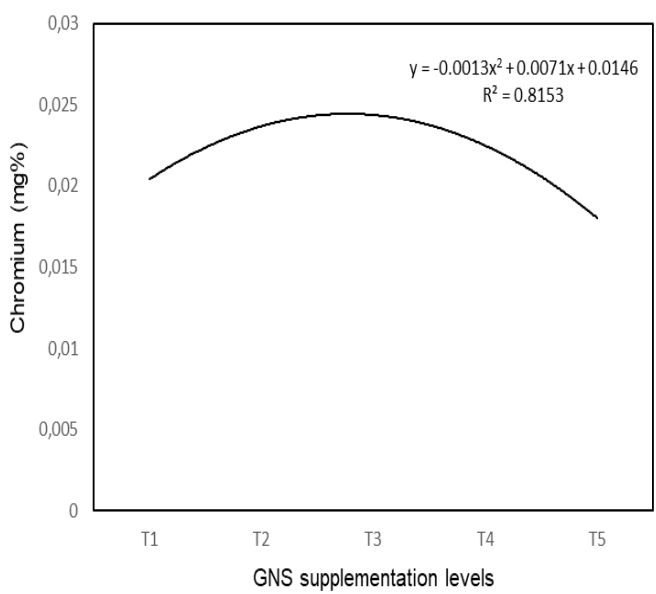

Figure 21. Estimation curve of the optimal plasma Cr of unsexed mixed cattle breed fed basal diet supplemented with GNS

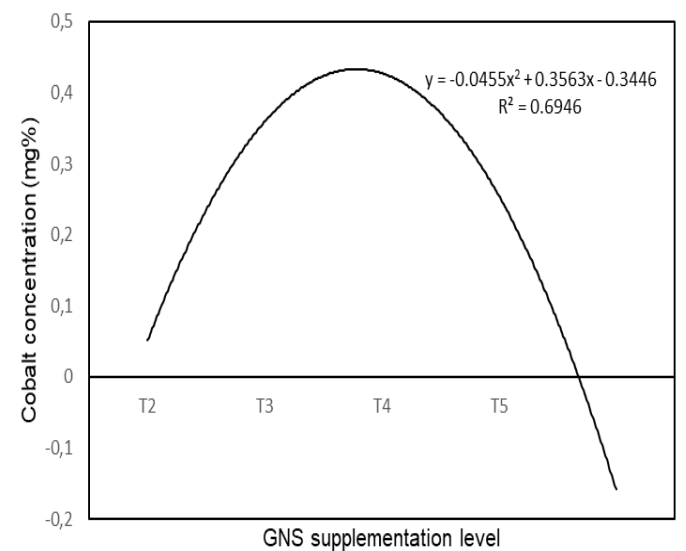

Figure 22. Estimation curve of the optimal plasma Co of unsexed mixed cattle breed fed basal diet supplemented with GNS 


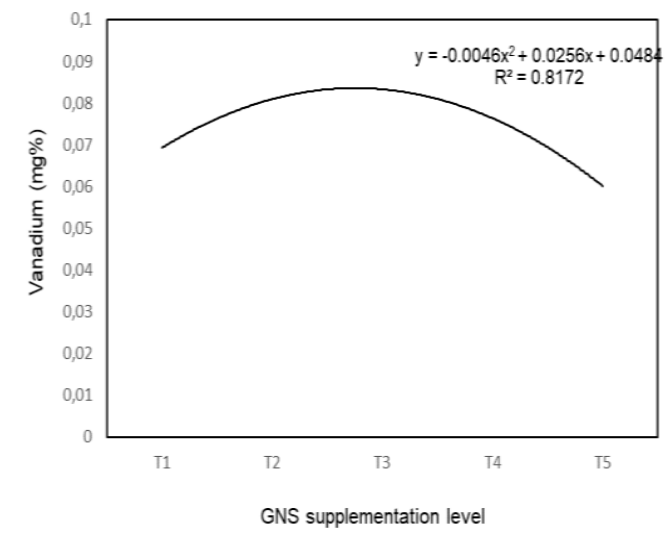

Figure 23. Estimation curve of the optimal plasma $V$ of unsexed mixed cattle breed fed basal diet supplemented with GNS

\section{Discussion}

\section{Nutrient composition of basal diet and GNS}

Studies have shown that biophysical and biochemical fractions are employed in the determination of uptake and availability of nutrients in a test feedstuff (Okoli et al., 2009). The feed value of feedstuff a function of its nutrient content, digestibility and palatability and consequently its associative effects with other feeds (Smith, 1992). Therefore, a proper understanding of the biophysical and biochemical fractions in all feed ingredients is essential (Okoli et al., 2009). The results of the nutrient biochemical component of GNS showed that GNS is rich in fibre but moderate in ash and protein. The $2.5 \%$ reported for ash in the present experiment is in harmony with the earlier findings of Abdulrazak et al. (2014) who observed a similar value of $2.5 \%$, but lower than $4.26 \%$ reported by PereaMoreno et al. (2018), 5.3\% reported by Khan et al. (2017) and 7.79\% reported by Akinfemi et al. (2012) for groundnut shell. The observed discrepancy in these results could be attributed in part to analytical methods adopted or soil type where the crop was harvested. The presence of fibre and ash (minerals) in groundnut shells imply that GNS could be used as a source fibre and mineral supplement for ruminants. The low moisture content of the GNS samples revealed that they can be easily dried to reduce the incidence of microbial attacks. Results of proximate biochemical analysis of GNS showed that crude protein (11.67\%) was higher than the range of $4.43-7.39 \%$ reported by other investigators (Akinfemi et al., 2012; Abdulrazak et al., 2014).

The moisture content $(8.8 \%)$ reported in the current study was slightly lower than $5.97 \%$ obtained for GNS by Perea-Moreno et al. (2018). The carbohydrate value $(35.33 \%)$ was higher than the range of 18.10 - $25.57 \%$ reported by Abdulrazak et al. (2014) and Khan et al. (2017), while the ether extract content (1.50\%) was slightly lower than $2.00 \%$ and $6.31 \%$ obtained by Khan et al. (2017) and Akinfemi et al. (2012), respectively. The crude fibre content reported in the present study was $17 \%$ lower and $47 \%$ higher than the values of $59.0 \%$ and $26.15 \%$ earlier reported by Abdulrazak et al. (2014) and Akinfemi et al. (2012), respectively in a similar experiment for groundnut shells. The disparity in the proximate results of the present study with the results of other authors may be due to environment and soil type differences, which has been reported to affect the nutrient composition of feedstuffs (Mecha and Adegbola, 1980; Melesse et al., 2012; Ogunbosoye et al., 2015). Standard deviation (SD) values across the values 
analyzed were narrow showing reliability of these mean values as reference values for the groundnut shell.

The results of the proximate composition of the basal diet (blue buffalo grass) as shown in Table 4.1 revealed that blue buffalo grass is an excellent source of nutrient (fibre, protein and energy) but low in ash content. Published data on the proximate composition of blue buffalo grass is lacking in the literature, hence there is no data to compare our findings with. The ash value $(1.51 \%)$ recorded in the present study for blue buffalo grass (Cenchrus ciliaris) was lower than $7.54-7.71 \%$ and $2.0-18.18 \%$ reported for Napier grass (Pennisetum purpureum) in South Africa and Nigeria by Rambau et al. (2016) and Okaraonye and Ikewuchi (2009), respectively. The choice of comparing our results with Napier grass in this study instead of other grasses used in ruminant feeding can be explained by the fact that it is high in nutrients (Rambau et al., 2016). The results of crude protein and ether extract value recorded in the present analysis for blue buffalo grass were lower than $13.16-15.80 \%$ crude protein and $3.25-3.63 \%$ ether extract reported by Rambau et al. (2016). The disparities in nutrient values could be linked to their genetic make-up. Therefore, forages with higher crude protein content are more nutritive. The moderate fibre level in blue buffalo grass is an indication that it can be easily digested and utilized efficiently by the rumen microbes. Standard deviation values across the values analyzed were narrow showing reliability of these mean values as reference values for the blue buffalo grass.

\section{Mineral composition of basal diet and GNS}

Minerals are vital nutrients with specific functions required in small amounts in the body (Gafar and Itodo, 2011) and their absence in the diet can lead to a significant reduction in growth and reproduction (McDonald et al., 1995; Gafar and Itodo, 2011). Generally, ash content gives an indication of the minerals present in feed material. The current study revealed that groundnut shells contain an appreciable quantity of ash an indication that it can serve as a source of mineral for ruminants. Aspects of the mineral profile of groundnut shell reported in the present study were higher than $0.042 \mathrm{~g} / 100 \mathrm{~g}$ $\mathrm{Na}, 0.705 \mathrm{~g} / 100 \mathrm{~g} \mathrm{~K}, 0.039 \mathrm{~g} / 100 \mathrm{~g} \mathrm{Mg}, 0.023 \mathrm{~g} / 100 \mathrm{~g} \mathrm{Ca}$ and $0.011 \mathrm{~g} / 100 \mathrm{~g} \mathrm{P}$ and lower than $6.97 \mathrm{mg} / 100 \mathrm{~g}$ iron and $3.20 \mathrm{mg} / 100 \mathrm{~g}$ zinc recorded by Atasie et al. (2009). The $\mathrm{Cu}$ $(2.67 \mathrm{mg} / 100 \mathrm{~g}), \mathrm{Zn}(5.04 \mathrm{mg} / 100 \mathrm{~g})$ and $\mathrm{Fe}(0.32 \mathrm{mg} / 100 \mathrm{~g})$ were higher than $0.002 \mathrm{mg} / 100 \mathrm{~g}, 0.003 \mathrm{mg} / 100 \mathrm{~g}$ and $0.115 \mathrm{mg} / 100 \mathrm{~g}$, respectively, reported by Grandawa (2014). Slight variations were noticed between the values recorded in the present study for $\mathrm{Ca}, \mathrm{P}, \mathrm{Mg}, \mathrm{Na}$ and $\mathrm{K}$ and the values of $0.644 \mathrm{~g} / 100 \mathrm{~g}, 0.134 \mathrm{~g} / 100 \mathrm{~g}, 0.253 \mathrm{~g} / 100 \mathrm{~g}$, $0.004 \mathrm{~g} / 100 \mathrm{~g}$ and $0.782 \mathrm{~g} / 100 \mathrm{~g}$ obtained for the same parameters by Akinfemi et al. (2012) for GNS. The results show that the values returned for $\mathrm{Ca}, \mathrm{Na}, \mathrm{K}, \mathrm{Mg}, \mathrm{P}, \mathrm{Zn}$ and $\mathrm{Cu}$ obtained in this research for blue buffalo grass were higher than $0.34,0.06,1.74,0.02$, $0.012 \mathrm{~g} / 100 \mathrm{~g}, 1.77 \mathrm{mg} / 100 \mathrm{~g}$, and $0.77 \mathrm{mg} / 100 \mathrm{~g}$ recorded for the same parameters in Napier grass (Rambau et al., 2016). However, the value recorded for $\mathrm{Mn}$ and Fe in the present study was far lower than $17.3 \mathrm{mg} / 100 \mathrm{~g}$ and $63.21 \mathrm{mg} / 100 \mathrm{~g}$ returned for the same parameters in Napier grass (Rambau et al., 2016). The disparity may be partly linked soil type (Zewdu et al., 2003) and genetic differences.

\section{$L W, A D G, B C S$ and blood mineral value of cattle}

The investigation revealed that cattle on diet T3 had improved LW, ADG and BCS. The improved LW, ADG and BCS in cattle fed diet T3 could partly be explained by enhanced utilization of the diet. Results revealed that GNS may have ability as a feed 
supplement for confined communal cattle. This is an agreement with the earlier reports of Atasie et al. (2009) and Abdulrazak et al. (2014) that GNS is moderate in essential nutrients. Blood samples are often analyzed with the aim of ascertaining the mineral status of animals. Blood is used as an index of animal nutritional status due to problems in determining mineral status from diet evaluation (Ogbuewu et al., 2015). The biologic availability of dietary minerals is usually at variance and hard to predict due to mineral availability can be influenced by chemical form and source of the mineral as well as the interactions among dietary constituents. The significantly high plasma $\mathrm{Ca}$ and $\mathrm{Na}$ obtained in indigenous cattle fed diets T2, T3, T4 and T5 indicate the high ability of the experimental animals to absorb these minerals from the feed. Phosphorus, the second most abundant mineral in the body after calcium is required for energy production, skeletal development and maintenance, cell growth and differentiation, muscle tissue building, membrane formation and maintenance of osmotic and acid-base balance (Suttle, 2010). The improved plasma phosphorus level recorded in indigenous cattle on diets T3, T4 and T5 is a pointer that experimental diets are not deficient in dietary phosphorus. This also means that diets T3, T4 and T5 are rich in phosphorus. The significantly increased plasma $\mathrm{Mg}$ and $\mathrm{K}$ level in cattle fed diets $\mathrm{T} 3, \mathrm{~T} 4$ and $\mathrm{T} 5$ is an indication that magnesium and potassium level in the diet is not compromised. This further supports the results of our mineral analysis which revealed that groundnut shells and basal diet are rich in macro-minerals. Increased chloride level for the group in diets T3 and T4 shows that the level of this mineral in the treatment diets are not the same to that of the control, meaning that diets T3 and T4 are high in chloride. The significantly increased value of plasma sulfur in the cattle fed diet T3 compared to the control and the groups fed diets T2, T4 and T5 could be attributed to diet, source and chemical form of the mineral, and interactions among dietary constituents which have been reported to influence blood mineral composition (Suttle, 2010).

Utilization of blood metabolite provides an immediate indication of an animal's nutritional status and is recommended when assessing the effects of a diet on the performance of animal in the short to medium term (Pambu-Gollah et al., 2000). Blood serum mineral and electrolyte values in the current study were unaffected by the dietary treatments. The micro-mineral analysis revealed that the $\mathrm{Mn}, \mathrm{Fe}, \mathrm{Cu}, \mathrm{C}, \mathrm{Cr}$, $\mathrm{Co}$ and $\mathrm{V}$ level of the indigenous cattle progressively increased with increasing levels of groundnut shell supplementation up to $700 \mathrm{~g} / \mathrm{kg}$ feed (diet T3) and started decreasing before it started falling. $\mathrm{Cu}, \mathrm{Zn}, \mathrm{Fe}$ and $\mathrm{Se}$ concentrations recorded in the present experiment were below the reference value of $0.8 \mathrm{mg} \%$ (copper and zinc), iron $(1.3 \mathrm{mg} \%$ ) and $0.08 \mathrm{mg} \%$ (Se) reported for a healthy finishing cattle (Davy et al., 2019). In contrast, the Mn value obtained in this experiment was higher than the values of $0.006 \mathrm{mg} \%$ reported for healthy cattle by Davy et al. (2019) in California, USA. The statistically increased plasma $\mathrm{Mn}, \mathrm{Fe}, \mathrm{Cu}$ and Cr concentrations obtained in group fed diet $\mathrm{T} 3$ compared to the cattle in the control group (T1) indicate better utilization of the diet. The observed variations on the blood mineral values recorded in this experiment with the values reported by Davy et al. (2019) in the USA could be attributed in part to soil type, breed and sex of the animals used.

\section{Optimization model}

Data on the use quadratic regression model to ascertain the optimal GNS supplementation level that optimize growth performance indices and blood mineral traits in cattle are not available. The results of the influence of GNS supplementation showed that GNS had a quadratic action on growth performance (LW, ADG and BCS) and plasma 
macro-minerals (Ca, P, Mg, K, Na, $\mathrm{Cl}$ and $\mathrm{S}$ ) and micro-minerals $(\mathrm{Mn}, \mathrm{Fe}, \mathrm{Cu}, \mathrm{C}, \mathrm{Cr}, \mathrm{C}$ and $\mathrm{V}$ ) with a $\mathrm{p}$ value of $<0.0001$ to $0.0002,0.0001$ to 0.0390 and $0.0001-0.0093$, respectively. LW, ADG and BCS were optimized at 315.88, 315.70 and $328.42 \mathrm{~g}$ GNS/ $\mathrm{kg}$ feed. The results of the plasma $\mathrm{Ca}, \mathrm{P}, \mathrm{Mg}, \mathrm{K}, \mathrm{Na}, \mathrm{Cl}$ and $\mathrm{S}$ were optimized at 319.05, $354.41,331.40,355.0,333.22,307.46$ and $288.60 \mathrm{~g}$ GNS/ $\mathrm{kg}$ feed, respectively. On the other hand, GNS supplementation at 299.04, 296.77, 345.74, 307.86, 273.08, 391.54 and $78.26 \mathrm{~g} / \mathrm{kg}$ feed optimized plasma $\mathrm{Mn}, \mathrm{Fe}, \mathrm{Cu}, \mathrm{C}, \mathrm{Cr}$, Co and V. Plasma Ca, P, Mg, K, $\mathrm{Na}, \mathrm{Cl}$ and $\mathrm{S}$ were optimized at varying GNS levels and the reasons for these variabilities is not fully known. However, this implied that cattle need varying levels of GNS supplemented diets to meet their mineral requirements. The values for coefficient of determination for growth performance indices were ranged $55.19 \%$ to $56.62 \%$, while macro-minerals were ranged $65.23 .9 \%$ to $80.86 \%$ which indicate high strength of GNS supplementation on plasma $\mathrm{Ca}, \mathrm{P}, \mathrm{Mg}, \mathrm{K}, \mathrm{Na}, \mathrm{Cl}$ and $\mathrm{S}$ in cattle using quadratic function. Also, the coefficient of determination values for plasma micro-minerals were ranged 53.76 - $81.72 \%$ except for plasma manganese which had $40.45 \%$. This may imply moderate strength of GNS supplementation on blood Mn concentration. The statistical optimization effect of GNS supplementation on blood macro-minerals and aspects of micro-minerals is a pointer that their values can be predicted at given supplementation level of GNS added in ration. The comparable changes in plasma Se and $\mathrm{Zn}$ may indicate that the influence of GNS supplemented diet was the same. However, further study on the effect of GNS supplementation in blood mineral parameters in cattle is desirable.

\section{Conclusion}

Groundnut shell is a rich source of dietary fibre and essential minerals and can be used as an excellent crude fibre and mineral sources in cattle diets. This is a pointer that GNS possesses the ability to mobilize and store important minerals in its shells. GNS supplementation influenced production parameters and blood mineral values in unsexed mixed cattle breed. GNS supplementation up to $700 \mathrm{~g} / \mathrm{kg}$ feed in cattle ration encouraged digestion and absorption of minerals from the digestive tracts. The low to a moderate coefficient of variation of mean blood mineral concentrations obtained in this study established the fact that these values could serve as standard blood mineral value for cattle fed groundnut shells supplemented the diet in the study area.

The optimal response pattern for production parameters (LW, ADG, BCS), blood macro-minerals $(\mathrm{Ca}, \mathrm{P}, \mathrm{Mg}, \mathrm{K}, \mathrm{Na}, \mathrm{Cl}, \mathrm{S})$ and aspect of blood micro-mineral ( $\mathrm{Mn}, \mathrm{Fe}$, $\mathrm{Cu}, \mathrm{C}, \mathrm{Cr}, \mathrm{Co}, \mathrm{V})$ parameters measured in unsexed mixed cattle breed were influenced by the groundnut shell supplementation. The results of the quadratic optimization model revealed that no single GNS rate optimized production data and blood minerals in the present study, meaning that groundnut shell supplementation level for optimal response depended on the production parameter and blood mineral parameter in question. Furthermore, the results of the optimization function revealed that GNS supplementation rate for optimal production parameters and blood minerals was achieved at a rate below $450 \mathrm{~g} / \mathrm{kg}$ feed. Hence, the feeding program for optimal production parameters and blood mineral levels in unsexed mixed cattle breed must consider the parameter under consideration. However, further research is needed to ascertain the influence of sex on the production indices and blood mineral profiles of unsexed mixed cattle breed under confined system fed BBG supplemented with GNS during the winter season in North West province, South Africa. 


\section{REFERENCES}

[1] Abdulrazak, S., Otie, D., Oniwapele, Y. A. (2014): Proximate analysis and anti-nutritional factors of groundnut and melon husk. - Online J. Anim. Feed Res. 4(2): 25-28.

[2] Akinfemi, A., Adua, M. M., Adu, O. A. (2012): Evaluation of nutritive values of tropical feed sources and by-products using in vitro gas production technique in ruminant animals. - Emir. J. Food Agric. 24(4): 348-353.

[3] AOAC (2002): Association of Official Analytical Chemists. Official Methods of Analysis. $-15^{\text {th }}$ ed. Washington DC, USA.

[4] Atasie, V. N., Akinhanmi, T. F., Ojiodu, C. C. (2009): Proximate analysis and physicochemical properties of groundnut (Arachis hypogaea L.). - Pak. J. Nut. 8(2): 194-197.

[5] Davis, J. P., Dean, L. L. (2016): Peanut composition, flavor and nutrition. - In: Stalker, H. T., Wilson, R. F. (eds.) Peanuts Genetics, Processing, and Utilization. AOCS Press, pp. 289-345.

[6] Davy, J. S., Forero, L. C., Shapero, M. W. K., Rao, D. R., Becchetti, T. A., Rivers, C. K., Stackhouse, J. W., DeAtley, L. K., McNabb, B. R. (2019): Mineral status of California beef cattle. - Anim. 3: 66-73.

[7] Gafar, M. K., Itodo, A. U. (2011): Proximate and mineral composition of hairy indigo leaves. - Electronic J. Environmental, Agric. and Food Chem. 10(3): 2007-2018.

[8] Grandawa, M. M. (2014): Characterization of the physicochemical properties of Arachis hypogea L. shells (groundnut) as environmental remediation. - International Conference on Chemical, Biological, and Environmental Sciences (ICCBES'14) May 12-13, 2014 Kuala Lumpur (Malaysia), pp. 1-10.

[9] Khan, M. T., Khan, M. I., Raza, S. H. A., Adnan, M., Khan, R., Hosseini, S. M., Syed, S. F., Shah, S. K. A., Khan, M. A., Ahmad, A. (2017): Effect of urea treated groundnut shells on feed intake, digestibility, nitrogen retention and economic value in growing rabbits. Int. J. Poult. and Fisheries Sci. 1(1): 1-7.

[10] Malau-Aduli, B. S., Eduvie, L., Lakpini, C., Malau-Aduli, A. E. O. (2003): Chemical compositions, feed intakes and digestibility of crop residues based on rations in nonlactating red Sokoto goats in the sub humid zone of North Nigeria. - Anim. Sci. J. 74: 8994.

[11] McDonald, P., Edward, R. A., Greenhalti, F. D., Morgan, C. A. (1995): Animal Nutrition. - Prentices Hall, London, pp. 101-122.

[12] Mecha, I., Adegbola, T. A. (1980): Chemical composition of some southern Nigeria forage eaten by goats. - In: Lehouerou, H. N. (ed.) Browse in Africa; the current state of knowledge. International Livestock Centre for Africa (ILCA), Addis Ababa, Ethiopia, pp. 303-306.

[13] Melesse, A., Steingass, H., Boguhn, J., Schollenberger, M., Rodehutscord, M. (2012): Effects of elevation and season on nutrient composition of leaves and green pods of Moringa stenopetala and Moringa oleifera. - Agroforest Syst. 86: 505-18.

[14] Mokolopi, B. G., Beighle, D. E. (2006): Evaluation of the mineral status of the communal grazing in the North West Province of South Africa. - J. South Afr. Vet. Assoc. 77(4): 179183.

[15] Ogbuewu, I. P., Ahiwe, E. U., Okoli, I. C., Iloeje, M. U. (2015): Interactions between Dietary Minerals and Reproduction in farm Animal. - Global J. Anim. Scientific Res. 3(2): 524-535.

[16] Ogunbosoye, D. O., Tona, G. O., Otukoya, F. K. (2015): Evaluation of the nutritive value of selected browse plant species in the southern guinea savannah of Nigeria for feeding to ruminant animals. - Brit. J. Appld. Sci. and Tech. 7(4): 386-395.

[17] Okaraonye, C. C., Ikewuchi, J. C. (2009): Nutritional and antinutritional components of Pennisetum purpureum (Schumach). - Pak. J. Nut. 8: 32-34. 
[18] Okoli, I. C., Omede, A. A., Ogbuewu, I. P., Uchegbu, M. C. (2009): Physical characteristics as indicators of poultry feed quality: A review. - Proc of the $3^{\text {rd }}$ Nig Int Poult Conf, February 22-26, 2009, Abeokuta, Ogun State, Nigeria, pp. 124-128.

[19] Pambu-Gollah, R., Cronje, P. B., Casey, N. H. (2000): An evaluation of the use of blood metabolite concentrations as indicators of nutritional status in free-ranging indigenous goats. - South Afr. J. Anim. Sci. 30: 115-120.

[20] Pauzenga, U. (1985): Feeding parent stock. - Zootech Int, pp. 22-25.

[21] Perea-Moreno, M., Manzano-Agugliaro, F., Hernandez-Escobedo, Q., Perea-Moreno, A. (2018): Peanut shell for energy: Properties and its potential to respect the environment. Sustainability 10: 3254. DOI:10.3390/su10093254.

[22] Pogge, D. J., Richter, E. L., Drewnoski, M. E., Hansen, S. L. (2012): Mineral concentrations of plasma and liver after injection with a trace mineral complex differ among Angus and Simmental cattle. - J. Anim. Sci. 90: 2692-2698.

[23] Rambau, M. D., Fushai, F., Baloyi, J. J. (2016): Productivity, chemical composition and ruminal degradability of irrigated Napier grass leaves harvested at three stages of maturity. - South Afr. J. Anim. Sci. 46(4): 398-408.

[24] Richter, E. L., Drewnoski, M. E., Hansen, S. L. (2012): Effects of increased dietary sulfur on beef steer mineral status, performance and meat fatty acid composition. - J. Anim. Sci. 90: 3945-3953.

[25] SAS (2010): SAS user guide: statistical version. - SAS institute Inc. Cary, NC USA. Version 9.3.

[26] Smith, O. B. (1992): Small ruminant feeding systems for small scale farmers in humid West Africa. - In: Stares, J. E. S., Said, A. N., Kategile, J. A. (eds.) The complementarity of feed resources for animal production in Africa. Proceedings of the Joint Feed Resources Networks Workshop held in Gaborone, Botswana, 4-8 March 1991. Africa Feeds Research Network. International Livestock Centre for Africa, Addis Ababa, Ethiopia, pp. 363-376.

[27] Suttle, N. F. (2010): Mineral Nutrition of Livestock. $-4^{\text {th }}$ ed. CABI Publishing, New York. [28] van Doosselaere, P. (2013): Production of oils. - In: Hamm, W., Hamilton, R. J., Calliauw, G. (eds.) Edible oil processing. Wiley Blackwell, Oxford, UK, pp. 55-96.

[29] Wildman, E. F., Jones, G. M., Wagner, P. E., Boman, R. L., Troutt, H. F., Lesch, T. N. (1982): A dry cow body condition scoring system and its relationship to selected production characteristics. - J. Dairy Sci. 65: 495-501.

[30] Zewdu, T., Baars, R. M. T., Yami, A. (2003): Effect of plant height at cutting and fertilizer on growth of Napier grass (Pennisetum purpureum). - Trop. Sci. J. 43(1): 57-61. 